\title{
FIXED POINT VARIATIONAL SOLUTIONS FOR UNIFORMLY CONTINUOUS PSEUDOCONTRACTIONS IN BANACH SPACES
}

\author{
ANIEFIOK UDOMENE
}

Received 27 June 2005; Revised 21 November 2005; Accepted 28 November 2005

Let $E$ be a reflexive Banach space with a uniformly Gâteaux differentiable norm, let $K$ be a nonempty closed convex subset of $E$, and let $T: K \rightarrow K$ be a uniformly continuous pseudocontraction. If $f: K \rightarrow K$ is any contraction map on $K$ and if every nonempty closed convex and bounded subset of $K$ has the fixed point property for nonexpansive self-mappings, then it is shown, under appropriate conditions on the sequences of real numbers $\left\{\alpha_{n}\right\},\left\{\mu_{n}\right\}$, that the iteration process $z_{1} \in K, z_{n+1}=\mu_{n}\left(\alpha_{n} T z_{n}+\left(1-\alpha_{n}\right) z_{n}\right)+$ $\left(1-\mu_{n}\right) f\left(z_{n}\right), n \in \mathbb{N}$, strongly converges to the fixed point of $T$, which is the unique solution of some variational inequality, provided that $K$ is bounded.

Copyright (C) 2006 Aniefiok Udomene. This is an open access article distributed under the Creative Commons Attribution License, which permits unrestricted use, distribution, and reproduction in any medium, provided the original work is properly cited.

\section{Introduction}

Let $E$ be a real Banach space with dual $E^{*}$ and $K$ a nonempty closed convex subset of $E$. Let $J: E \rightarrow 2^{E^{*}}$ denote the normalized duality mapping defined by $J(x):=\left\{f \in E^{*}\right.$ : $\left.\langle x, f\rangle=\|x\|^{2},\|f\|=\|x\|, x \in E\right\}$ where $\langle\cdot, \cdot\rangle$ denotes the generalized duality pairing. Following Morales [6], a mapping $T$ with domain $D(T)$ and range $\mathscr{R}(T)$ in $E$ is called strongly pseudocontractive if for some constant $k<1$ and $\forall x, y \in D(T)$,

$$
(\lambda-k)\|x-y\| \leq\|(\lambda I-T)(x)-(\lambda I-T)(y)\|
$$

for all $\lambda>k$; while $T$ is called a pseudocontraction if (1.1) holds for $k=1$. The mapping $T$ is called Lipschitz if there exists $L \geq 0$ such that $\|T x-T y\| \leq L\|x-y\|, \forall x, y \in D(T)$. The mapping $T$ is called nonexpansive if $L=1$ and is called a (strict) contraction if $L<1$. Every nonexpansive mapping is a pseudocontraction. The converse is not true. The example, $T(x)=1-x^{2 / 3}, 0 \leq x \leq 1$, is a continuous pseudocontraction which is not nonexpansive. It follows from a result of Kato [3] that $T$ is pseudocontractive if and only if there exists $j(x-y) \in J(x-y)$ such that $\langle T x-T y, j(x-y)\rangle \leq\|x-y\|^{2}, \forall x, y \in D(T)$. 
In [9], Schu introduced the iterative process (1.2) below and proved the following theorem.

Theorem 1.1 [9, Theorem 2.4, page 113]. Let $K$ be a nonempty, closed convex, and bounded subset of a Hilbert space $H$; let $T: K \rightarrow K$ be a Lipschitz pseudocontractive map with Lipschitz constant $L \geq 0 ;\left\{\lambda_{n}\right\}_{n \in \mathbb{N}} \subset(0,1)$ with $\lim _{n \rightarrow \infty} \lambda_{n}=1 ;\left\{\alpha_{n}\right\}_{n \in \mathbb{N}} \subset(0,1)$ with $\lim _{n \rightarrow \infty} \alpha_{n}=0$ such that $\left(\left\{\alpha_{n}\right\},\left\{\mu_{n}\right\}\right)$ has property $(A),\left\{\left(1-\mu_{n}\right)\left(1-\lambda_{n}\right)^{-1}\right\}$ is bounded, and $\lim _{n \rightarrow \infty}\left(1-\mu_{n}\right) / \alpha_{n}=0$, where $k_{n}:=\left(1+\alpha_{n}^{2}(1+L)^{2}\right)^{1 / 2}$ and $\mu_{n}:=\lambda_{n} / k_{n}$, for all $n \in \mathbb{N}$; fix an arbitrary point $w \in K$, and define that for all $n \in \mathbb{N}$,

$$
z_{n+1}:=\mu_{n+1}\left(\alpha_{n} T z_{n}+\left(1-\alpha_{n}\right) z_{n}\right)+\left(1-\mu_{n+1}\right) w .
$$

Then $\left\{z_{n}\right\}_{n}$ converges strongly to the unique fixed point of $T$ closest to $w$.

Here the pair of sequences $\left(\left\{\alpha_{n}\right\}_{n},\left\{\mu_{n}\right\}_{n}\right) \subset(0, \infty) \times(0,1)$ is said to have property (A) if and only if the following conditions hold.

(i) $\left\{\alpha_{n}\right\}_{n}$ is decreasing;

(ii)' $\left\{\mu_{n}\right\}_{n}$ is strictly increasing;

(iii)' There exists a strictly increasing sequence $\left\{\beta_{n}\right\}_{n} \subset \mathbb{N}$ such that
(a) ${ }^{\prime} \lim _{n}\left(\alpha_{n}-\alpha_{n+\beta_{n}}\right) /\left(1-\mu_{n}\right)=0$;
(b) $\lim _{n}\left(1-\mu_{n+\beta_{n}}\right)\left(1-\mu_{n}\right)^{-1}=1$;
(c) $\lim _{n} \beta_{n}\left(1-\mu_{n}\right)=\infty$.

The first iterative process of this nature was introduced by Halpern [2]: for any fixed $w \in K$ and arbitrary $z_{0} \in K$,

$$
z_{n+1}=\mu_{n} T z_{n}+\left(1-\mu_{n}\right) w, \quad n=0,1,2, \ldots
$$

where $\left\{\mu_{n}\right\}$ is a sequence in $(0,1)$ with $\lim _{n \rightarrow \infty} \mu_{n}=1$.

In [8], Moudafi proposed a viscosity approximation method of selecting a particular fixed point of a given nonexpansive mapping in Hilbert spaces, where he proved the following theorem.

Theorem 1.2 [8, Theorem 2.2, page 48]. Let $H$ be a Hilbert space, let $T: K \rightarrow K$ be a nonexpansive self-mapping of a nonempty closed convex subset $K$ of $H$, and let $f: K \rightarrow K$ be a contraction. With an initial $z_{0} \in K$, define the sequence $\left\{z_{n}\right\}$ by

$$
z_{n+1}=\frac{1}{1+\epsilon_{n}} T z_{n}+\frac{\epsilon_{n}}{1+\epsilon_{n}} f\left(z_{n}\right)
$$

Supposed that $\lim _{n \rightarrow \infty} \epsilon_{n}=0, \sum_{n=1}^{\infty} \epsilon_{n}=\infty$, and $\lim _{n \rightarrow \infty}\left|1 / \epsilon_{n+1}-1 / \epsilon_{n}\right|=0$. Then $\left\{z_{n}\right\}$ converges strongly to the unique solution of the variational inequality:

$$
\text { find } \tilde{x} \in F(T) \text { such that }\langle(I-f) \tilde{x}, \tilde{x}-x\rangle \leq 0, \quad \forall x \in F(T) \text {, }
$$

(i.e., the unique solution of the operator $\operatorname{Proj}_{F(T)} \circ f$ ).

$\mathrm{Xu}$ [12] extended Theorem 1.2 to the more general uniformly smooth Banach spaces. If $\Pi_{K}$ denotes the set of all contractions on $K$, he proved the following theorem. 
Theorem 1.3 [12, Theorem 4.2, page 289]. Let E be a uniformly smooth Banach space, $K$ a closed convex subset of $E$, and $T: K \rightarrow K$ a nonexpansive mapping with $F(T) \neq \varnothing$, and $f \in \Pi_{K}$. Assume that $\left\{\alpha_{n}\right\} \subset(0,1)$ satisfies the following conditions:

(i) $\lim _{n \rightarrow \infty} \alpha_{n}=0$;

(ii) $\sum_{n=0}^{\infty} \alpha_{n}=\infty$;

(iii) either $\lim _{n \rightarrow \infty} \alpha_{n+1} / \alpha_{n}=1$ or $\sum_{n=0}^{\infty}\left|\alpha_{n+1}-\alpha_{n}\right|<\infty$.

Then the sequence $\left\{z_{n}\right\}$ generated by $z_{0} \in K$,

$$
z_{n+1}:=\alpha_{n} f\left(z_{n}\right)+\left(1-\alpha_{n}\right) T z_{n}, \quad n=0,1,2, \ldots,
$$

converges strongly to $Q(f)$, where $Q: \Pi_{K} \rightarrow F(T)$ is defined by $Q(f):=\sigma-\lim _{t \rightarrow 0} x_{t}$, with $x_{t}$ satisfying

$$
x_{t}=t T x_{t}+(1-t) f\left(x_{t}\right) \text {. }
$$

Let $K$ be a nonempty closed convex and bounded subset of a real reflexive Banach space with a uniformly Gâteaux differentiable norm. Further to Theorems 1.2 and 1.3, the purpose of this paper is to use the following iteration process: $z_{1} \in K$,

$$
z_{n+1}=\mu_{n}\left(\alpha_{n} T z_{n}+\left(1-\alpha_{n}\right) z_{n}\right)+\left(1-\mu_{n}\right) f\left(z_{n}\right), \quad n \in \mathbb{N},
$$

where $\left\{\mu_{n}\right\}_{n},\left\{\alpha_{n}\right\}_{n}$ are sequences in $(0,1)$ and $f: K \rightarrow K$ is a contraction map, to approximate the fixed point of a uniformly continuous pseudocontraction, which solves some variational inequality. If the map $f$ is a constant map then we recover the iteration process (1.2) from (1.8).

\section{Preliminaries}

Let $E$ be a real normed linear space and let $S:=\{x \in E:\|x\|=1\}$. $E$ is said to have a Gâteaux differentiable norm and $E$ is called smooth if the limit

$$
\lim _{t \rightarrow 0} \frac{\|x+t y\|-\|x\|}{t}
$$

exists for each $x, y \in S$. E is said to have a uniformly Gâteaux differentiable norm if for each $y \in S$ the limit is attained uniformly for $x \in S$.

The modulus of smoothness of $E$ is defined by

$$
\rho_{E}(\tau):=\sup \left\{\frac{\|x+y\|+\|x-y\|}{2}-1:\|x\|=1,\|y\|=\tau\right\}, \quad \tau>0 .
$$

$E$ is equivalently said to be smooth if $\rho_{E}(\tau)>0 \forall \tau>0$. Every uniformly smooth Banach space is a reflexive Banach space with a uniformly Gâteaux differentiable norm. An example given in [7] illustrates that this inclusion is proper.

Let $E$ be a linear space and let $K$ be a subset of $E$. Then, for any $x \in K$, the set $I_{K}(x)=$ $\{x+\lambda(z-x): z \in K, \lambda \geq 1\}$ is called the inward set of $x$. A mapping $T: K \rightarrow E$ is said to satisfy the inward condition if $T x \in I_{K}(x)$ for each $x \in K$, and is said to satisfy the weakly inward condition if $T x \in c l\left[I_{K}(x)\right]$, the closure of $I_{K}(x)$, for each $x \in K$. 
We will let $\operatorname{LIM}_{n}$ be a Banach limit. Recall that $\operatorname{LIM}_{n} \in\left(\ell^{\infty}\right)^{*}$ such that $\left\|\operatorname{LIM}_{n}\right\|=1$, $\liminf _{n \rightarrow \infty} a_{n} \leq \operatorname{LIM}_{n} a_{n} \leq \limsup \sup _{n \rightarrow \infty} a_{n}$, and $\operatorname{LIM}_{n}=a_{n} \operatorname{LIM}_{n} a_{n+1}$ for all $\left\{a_{n}\right\}_{n} \in \ell^{\infty}$.

The modulus of uniform continuity, $\delta(\epsilon)$, of $T$ is defined for all $\epsilon>0$ by

$$
\delta(\epsilon)=\sup \{\lambda:\|x-y\|<\lambda \Longrightarrow\|T x-T y\|<\epsilon\}
$$

and $\delta(0)=0$. By [4, Proposition 3], $\delta(\epsilon)$ is nondecreasing, $0 \leq \delta(\epsilon) \leq \infty$, and $\delta(\| T x-$ $T y \|) \leq\|x-y\|$, for all $x, y \in E$. Furthermore, [4, Propositions 1 and 2] assert that the function

$$
\phi(t)=\sup \{s: \delta(s) \leq t\}
$$

called the pseudo-inverse of $\delta$ is nondecreasing and right continuous, $0 \leq \phi(t) \leq \infty$ for $t \geq 0$ and $\|T x-T y\| \leq \phi(\|x-y\|) \forall x, y \in E$.

The following lemmas will be needed in the sequel. Lemma 2.1 is well known, (see, e.g., [7]). The proof of Lemma 2.2 can be deduced from [11, Lemma 2.5].

Lemma 2.1. Let $E$ be an arbitrary real Banach space. Then

$$
\|x+y\|^{2} \leq\|x\|^{2}+2\langle y, j(x+y)\rangle
$$

for all $x, y \in E$ and for all $j(x+y) \in J(x+y)$.

LEMMA 2.2. Let $\left\{a_{n}\right\}_{n}$ be a sequence of nonnegative real numbers such that

$$
a_{n+1} \leq\left(1-\alpha_{n}\right) a_{n}+\alpha_{n} \beta_{n}, \quad n \in \mathbb{N},
$$

where $\left\{\alpha_{n}\right\}_{n} \subset[0,1],\left\{\beta_{n}\right\}_{n} \subset[0,1]$, and $\sum_{n=0}^{\infty} \alpha_{n}=\infty, \lim _{n \rightarrow \infty} \beta_{n}=0$. Then, $\lim _{n \rightarrow \infty} a_{n}=0$.

Lemma 2.3, Proposition 2.4, and Lemma 2.5 that follow appear in [10]. For completeness, we present also their proofs.

Lemma 2.3. Let $E$ be a Banach space. Suppose $K$ is a nonempty closed convex subset of $E$ and $T: K \rightarrow E$ is a continuous pseudocontraction satisfying the weakly inward condition. Then for each contraction map $f: K \rightarrow K$, with contraction constant $\alpha \in[0,1)$, there exists a unique continuous path $t \rightarrow x_{t} \in K, t \in[0,1)$ satisfying

$$
x_{t}=t T x_{t}+(1-t) f\left(x_{t}\right)
$$

Proof. Let $f: K \rightarrow K$ be a contraction map with constant $\alpha \in[0,1)$. Then, for each $t \in$ $[0,1)$, the mapping $T_{t}^{f}: K \rightarrow E$ defined by $T_{t}^{f}(x)=t T x+(1-t) f(x)$ is a continuous strong pseudocontraction with constant $t+(1-t) \alpha \in[0,1)$, which satisfies the weakly inward condition. By [1, Corollary 1$], T_{t}^{f}$ has a unique fixed point $x_{t} \in K$, that is,

$$
x_{t}=t T x_{t}+(1-t) f\left(x_{t}\right)
$$


To prove the continuity of the path, we follow the same line of arguments as in [7]. Let $t_{0} \in[0,1)$. Then for all $j\left(x_{t}-x_{t_{0}}\right) \in J\left(x_{t}-x_{t_{0}}\right)$,

$$
\begin{aligned}
\left\|x_{t}-x_{t_{0}}\right\|^{2}= & t\left\langle T x_{t}-T x_{t_{0}}, j\left(x_{t}-x_{t_{0}}\right)\right\rangle+(1-t)\left\langle f\left(x_{t}\right)-f\left(x_{t_{0}}\right), j\left(x_{t}-x_{t_{0}}\right)\right\rangle \\
& +\left(t-t_{0}\right)\left\langle T x_{t_{0}}-f\left(x_{t_{0}}\right), j\left(x_{t}-x_{t_{0}}\right)\right\rangle \\
\leq & (t+(1-t) \alpha)\left\|x_{t}-x_{t_{0}}\right\|^{2}+\left|t-t_{0}\right|\left\|T x_{t_{0}}-f\left(x_{t_{0}}\right)\right\|\left\|x_{t}-x_{t_{0}}\right\|,
\end{aligned}
$$

so that $\left\|x_{t}-x_{t_{0}}\right\| \leq\left(\left|t-t_{0}\right| /(1-t)(1-\alpha)\right)\left\|T x_{t_{0}}-f\left(x_{t_{0}}\right)\right\|$. Hence the proof.

Proposition 2.4. Let E be a Banach space and let $K$ be a nonempty closed convex subset of $E$. Let the mapping $T: K \rightarrow E$ be a pseudocontraction such that for each contraction map, $f: K \rightarrow K$ with contraction constant $\alpha \in[0,1)$, the equation

$$
x=t T x+(1-t) f(x)
$$

has a solution $x_{t}$ for every $t \in[0,1)$. Then the following hold.

(i) If for some $u \in K$, the path $y_{t}=t T y_{t}+(1-t) u$ is bounded, then for any contraction map $f: K \rightarrow K$, the path $\left\{x_{t}\right\}$ described by (2.7) is bounded.

(ii) If $T$ has a fixed point in $K$, then the path $\left\{x_{t}\right\}$ is bounded.

(iii) If $x^{*} \in F(T)$, then for all $j\left(x_{t}-x^{*}\right) \in J\left(x_{t}-x^{*}\right)$,

$$
\left\langle x_{t}-f\left(x_{t}\right), j\left(x_{t}-x^{*}\right)\right\rangle \leq 0 \text {. }
$$

(iv) If $0 \leq s \leq t<1$ then

$$
\left\|x_{t}-T x_{t}\right\| \leq \frac{1+\alpha}{1-\alpha}\left\|x_{s}-T x_{s}\right\|
$$

Proof. (i) Let the path $\left\{y_{t}\right\}$ given by $y_{t}=t T y_{t}+(1-t) u$, for some $u \in K$, be bounded. Then the set $\left\{f\left(y_{t}\right)\right\}$ is bounded. Let $j\left(x_{t}-y_{t}\right) \in J\left(x_{t}-y_{t}\right)$. From the estimates

$$
\begin{aligned}
\left\|x_{t}-y_{t}\right\|^{2} & =t\left\langle T x_{t}-T y_{t}, j\left(x_{t}-y_{t}\right)\right\rangle+(1-t)\left\langle f\left(x_{t}\right)-u, j\left(x_{t}-y_{t}\right)\right\rangle \\
& \leq t\left\|x_{t}-y_{t}\right\|^{2}+(1-t)\left\|f\left(x_{t}\right)-u\right\|\left\|x_{t}-y_{t}\right\|,
\end{aligned}
$$

we have that $\left\|x_{t}-y_{t}\right\| \leq\left\|f\left(x_{t}\right)-u\right\| \leq \alpha\left\|x_{t}-y_{t}\right\|+\left\|f\left(y_{t}\right)-u\right\|$. Thus,

$$
\left\|x_{t}-y_{t}\right\| \leq \frac{1}{1-\alpha}\left\|f\left(y_{t}\right)-u\right\|
$$

Hence, $\left\{x_{t}\right\}$ is bounded.

(ii) Let $x^{*} \in F(T)$, and let $j\left(x_{t}-x^{*}\right) \in J\left(x_{t}-x^{*}\right)$. Then

$$
\begin{aligned}
\left\|x_{t}-x^{*}\right\|^{2} & =t\left\langle T x_{t}-x^{*}, j\left(x_{t}-x^{*}\right)\right\rangle+(1-t)\left\langle f\left(x_{t}\right)-x^{*}, j\left(x_{t}-x^{*}\right)\right\rangle \\
& \leq t\left\|x_{t}-x^{*}\right\|^{2}+(1-t)\left\|f\left(x_{t}\right)-x^{*}\right\|\left\|x_{t}-x^{*}\right\|
\end{aligned}
$$


so that $\left\|x_{t}-x^{*}\right\| \leq\left\|f\left(x_{t}\right)-x^{*}\right\| \leq \alpha\left\|x_{t}-x^{*}\right\|+\left\|f\left(x^{*}\right)-x^{*}\right\|$. Thus,

$$
\left\|x_{t}-x^{*}\right\| \leq \frac{1}{1-\alpha}\left\|f\left(x^{*}\right)-x^{*}\right\| .
$$

Hence, $\left\{x_{t}\right\}$ is bounded.

(iii) Let $x^{*} \in F(T)$, and let $j\left(x_{t}-x^{*}\right) \in J\left(x_{t}-x^{*}\right)$. Then

$$
\begin{aligned}
\left\langle x_{t}-f\left(x_{t}\right), j\left(x_{t}-x^{*}\right)\right\rangle & =t\left\langle T x_{t}-f\left(x_{t}\right), j\left(x_{t}-x^{*}\right)\right\rangle=t\left\langle T x_{t}-x^{*}, j\left(x_{t}-x^{*}\right)\right\rangle \\
& +t\left\langle x^{*}-f\left(x_{t}\right), j\left(x_{t}-x^{*}\right)\right\rangle \leq t\left\langle x_{t}-f\left(x_{t}\right), j\left(x_{t}-x^{*}\right)\right\rangle .
\end{aligned}
$$

Thus, $\left\langle x_{t}-f\left(x_{t}\right), j\left(x_{t}-x^{*}\right)\right\rangle \leq 0$.

(iv) Let $0 \leq s \leq t<1$. Then

$$
\begin{aligned}
\left\|x_{t}-T x_{t}\right\| & =\frac{1-t}{t}\left\|x_{t}-f\left(x_{t}\right)\right\| \\
& \leq \frac{1-t}{t}\left[(1+\alpha)\left\|x_{t}-x_{s}\right\|+\frac{s}{1-s}\left\|x_{s}-T x_{s}\right\|\right] \\
& \leq \frac{1-t}{t}\left[\frac{(1+\alpha)(t-s)}{(1-\alpha)(1-t)(1-s)}+\frac{s}{1-s}\right]\left\|x_{s}-T x_{s}\right\| \\
& \leq \frac{(1+\alpha)(1-t)}{(1-\alpha) t}\left[\frac{t-s}{(1-t)(1-s)}+\frac{s}{1-s}\right]\left\|x_{s}-T x_{s}\right\| \\
& =\frac{1+\alpha}{1-\alpha}\left\|x_{s}-T x_{s}\right\| .
\end{aligned}
$$

Lemma 2.5. Let $E$ be a reflexive Banach space with a uniformly Gâteaux differentiable norm, let $K$ be a nonempty closed convex subset of $E$, let $T: K \rightarrow E$ be a continuous pseudocontraction satisfying the weakly inward condition, and let $f: K \rightarrow K$ be a contraction map with constant $\alpha \in[0,1)$. Suppose that every nonempty closed convex and bounded subset of $K$ has the fixed point property (f.p.p.) for nonexpansive self-mappings. If there exists $u_{0} \in K$ such that the set

$$
B=\left\{x \in K: T x=u_{0}+\lambda\left(x-u_{0}\right) \text { for some } \lambda>1\right\}
$$

is bounded, then the path $\left\{x_{t}\right\}, t \in[0,1)$ described by (2.7) converges strongly to the fixed point of $T$, which is the unique solution of the variational inequality

$$
p \in F(T) \text { such that }\left\langle p-f(p), j\left(p-x^{*}\right)\right\rangle \leq 0, \quad x^{*} \in F(T) .
$$

Proof. It follows from Lemma 2.3 that for each contraction map $f: K \rightarrow K$ there exists a unique continuous path $t \rightarrow x_{t} \in K, t \in[0,1)$ satisfying (2.7). Let there exists $u_{0} \in K$ such that the set $B$ is bounded. Then by Proposition 2.4(i), the path $\left\{x_{t}\right\}$ described by (2.7) is bounded. It is easy to see that this implies that the set $\left\{f\left(x_{t}\right): t \in[0,1)\right\}$ is 
bounded. The boundedness of the set $\left\{T x_{t}: t \in[0,1)\right\}$ follows from Proposition 2.4(iv). Let $\sup _{t \in[0,1)}\left\|x_{t}\right\| \leq M$. Then $\left\|x_{t}-x_{s}\right\| \leq 2 M$ for any $t, s \in[0,1)$. Set $x_{n}=x_{t_{n}}$ for $t_{n} \rightarrow$ $1^{-}$. Define $\psi: K \rightarrow \mathbb{R}$ by $\psi(x)=\operatorname{LIM}_{n}\left\|x_{n}-x\right\|^{2} \forall x \in K$. Since $E$ is reflexive, $\psi$ is convex, continuous and $\psi(x) \rightarrow \infty$ as $\|x\| \rightarrow \infty$, we have that the set $C:=\{y \in K: \psi(y)=$ $\left.\inf _{x \in K} \psi(x)\right\}$ is nonempty, closed and convex. We show that $C$ is bounded. Let $y \in C$. Then $\psi(y) \leq \underset{n}{\operatorname{LIM}}\left\|x_{n}-x_{0}\right\|^{2} \leq 4 M^{2}$, where $x_{0} \equiv x_{t_{0}}$. Applying the convexity of the functional $(1 / 2)\|\cdot\|^{2}: K \rightarrow \mathbb{R}$, we deduce that

$$
\|y\|^{2} \leq \underset{n}{2 \operatorname{LIM}}\left\|x_{n}-y\right\|^{2}+\underset{n}{2 \operatorname{LIM}}\left\|x_{n}\right\|^{2} \leq 2 \psi(y)+2 M^{2} \leq 10 M^{2},
$$

that is, $\|y\| \leq \sqrt{10} M, \forall y \in C$. Thus, $C$ is bounded. The mapping $J_{1}=(2 I-T)^{-1}$ is a nonexpansive self-mapping of $K$ (see [5, Theorem 6]). $C$ is invariant under $J_{1}$. Indeed, let $y \in C$. Then

$$
\begin{aligned}
\psi\left(J_{1}(y)\right) & =\underset{n}{\operatorname{LIM}}\left\|x_{n}-J_{1}(y)\right\|^{2} \leq \underset{n}{\operatorname{LIM}}\left(\left\|x_{n}-J_{1}\left(x_{n}\right)\right\|+\left\|x_{n}-y\right\|\right)^{2} \\
& \leq \operatorname{LIM}_{n}\left(\left\|x_{n}-T x_{n}\right\|+\left\|x_{n}-y\right\|\right)^{2}=\operatorname{LIM}_{n}\left\|x_{n}-y\right\|^{2}=\psi(y) .
\end{aligned}
$$

By hypothesis, $J_{1}$ has a fixed point $p \in C$. Thus, $T p=p$. Let $\tau \in(0,1)$. Then $\psi(p) \leq$ $\psi((1-\tau) p+\tau x), x \in K$, and using Lemma 2.1, we have that $0 \leq(\psi((1-\tau) p+\tau x)-$ $\psi(p)) / \tau \leq-2 \operatorname{LIM}_{n}\left\langle x-p, j\left(x_{n}-p-\tau(x-p)\right)\right\rangle$. Thus

$$
\operatorname{LIM}_{n}\left\langle x-p, j\left(x_{n}-p-\tau(x-p)\right)\right\rangle \leq 0
$$

Since, in this setting, $J$ is norm-to-weak* uniformly continuous on bounded sets, letting $\tau \rightarrow 0$, we have that

$$
\operatorname{LIM}_{n}\left\langle x-p, j\left(x_{n}-p\right)\right\rangle \leq 0, \quad x \in K
$$

In particular,

$$
\operatorname{LIM}_{n}\left\langle f(p)-p, j\left(x_{n}-p\right)\right\rangle \leq 0
$$

Observe that

$$
(1-\alpha)\left\|x_{n}-p\right\|^{2} \leq\left\langle x_{n}-f\left(x_{n}\right), j\left(x_{n}-p\right)\right\rangle+\left\langle f(p)-p, j\left(x_{n}-p\right)\right\rangle .
$$

Using Proposition 2.4(iii) and (2.25), we have find that $\operatorname{LIM}_{n}\left\|x_{n}-p\right\|=0$. Therefore, there exists a subsequence $\left\{x_{n_{k}}\right\}$ of $\left\{x_{n}\right\}$ such that $x_{n_{k}} \rightarrow p$ as $k \rightarrow \infty$. Assume that there is another subsequence $\left\{x_{n_{l}}\right\}$ of $\left\{x_{n}\right\}$ such that $x_{n_{l}} \rightarrow q \in F(T)$ as $l \rightarrow \infty$. With $x_{n_{k}} \rightarrow p$ and setting $x^{*}=q$, it follows from Proposition 2.4(iii) that

$$
\langle p-f(p), j(p-q)\rangle \leq 0
$$


Also, with $x_{n_{l}} \rightarrow q$ and setting $x^{*}=p$ in Proposition 2.4(iii), we have that

$$
\langle q-f(q), j(q-p)\rangle \leq 0 .
$$

Inequalities (2.27) and (2.28) yield that

$$
\|p-q\|^{2} \leq\langle f(p)-f(q), j(p-q)\rangle \leq \alpha\|p-q\|^{2},
$$

which implies that $p=q$, since $\alpha \in[0,1)$. Thus, $x_{n} \rightarrow p$ as $n \rightarrow \infty$ and $p \in F(T)$ is unique. Again, using Proposition 2.4(iii), we observe that

$$
\left\langle p-f(p), j\left(p-x^{*}\right)\right\rangle \leq 0, \quad \forall x^{*} \in F(T) .
$$

Hence, $p$ is the unique solution of the variational inequality (2.20). This concludes the proof of Lemma 2.5 .

\section{Main results}

In the results that follow, if the map $T$ is uniformly continuous and $\delta(\epsilon)$ denotes the modulus of continuity of $T$, we will let $\phi$ denote the pseudoinverse of $\delta$ and will assume that the set $\{\phi(t) / t: 0<t<1\}$ is bounded. Observe that if $T$ is Lipschitz, then it is clear that the set $\{\phi(t) / t: 0<t<1\}$ is bounded.

Theorem 3.1. Let $K$ be a nonempty closed convex and bounded subset of a real Banach space E. Let $T: K \rightarrow K$ be a uniformly continuous pseudocontraction and let $f: K \rightarrow K$ be a contraction map with contraction constant $\alpha \in[0,1)$. Let $\left\{z_{n}\right\}$ be a sequence generated from an arbitrary $z_{1} \in K$ by (1.8), where $\left\{\mu_{n}\right\},\left\{\alpha_{n}\right\}$ are real sequences in $(0,1)$ satisfying the following conditions:

(i) $\left\{\alpha_{n}\right\}$ is decreasing and $\lim _{n \rightarrow \infty} \alpha_{n}=0$;

(ii) $\lim _{n \rightarrow \infty} \mu_{n}=1$ and $\sum_{n=0}^{\infty}\left(1-\mu_{n}\right)=\infty$;

(iii) (a) $\lim _{n \rightarrow \infty}\left(1-\mu_{n}\right) / \alpha_{n}=0$,

(b) $\lim _{n \rightarrow \infty} \alpha_{n}^{2} /\left(1-\mu_{n}\right)=0$,

(c) $\lim _{n \rightarrow \infty}\left|\mu_{n}-\mu_{n-1}\right| /\left(1-\mu_{n}\right)^{2}=0$,

(d) $\lim _{n \rightarrow \infty}\left(\alpha_{n-1}-\alpha_{n}\right) / \alpha_{n-1}\left(1-\mu_{n}\right)=0$.

Then $\left\|z_{n}-T z_{n}\right\| \rightarrow 0$ as $n \rightarrow \infty$.

Proof. We first prove that $\left\|z_{n}-x_{n}\right\| \rightarrow 0$ as $n \rightarrow \infty$, where $\left\{x_{n}\right\}$ is a sequence satisfying (2.7).

Set $t_{n}=\alpha_{n} /\left(1-\mu_{n}+\alpha_{n}\right), \forall n \in \mathbb{N}$. Then $t_{n} \in(0,1)$ for each $n \in \mathbb{N}$. By the given condition (iii)(a), $t_{n} \rightarrow 1$ as $n \rightarrow \infty$. It follows from Lemma 2.3 that there exists a unique sequence $\left\{x_{n}\right\} \subset K$ satisfying the following conditions:

$$
x_{n}=t_{n} T x_{n}+\left(1-t_{n}\right) f\left(x_{n}\right), \quad n \in \mathbb{N} .
$$

Equation (3.1) can be rewritten as follows:

$$
x_{n}=\mu_{n}\left(\alpha_{n} T x_{n}+\left(1-\alpha_{n}\right) x_{n}\right)+\left(1-\mu_{n}\right) f\left(x_{n}\right)+\left(1-\mu_{n}\right) \alpha_{n}\left(T x_{n}-x_{n}\right) .
$$


Using the pseudocontractivity of $T$, we make the following estimates:

$$
\begin{aligned}
\left\|z_{n+1}-x_{n}\right\|^{2}= & \mu_{n} \alpha_{n}\left\langle T z_{n}-T x_{n}, j\left(z_{n+1}-x_{n}\right)\right\rangle+\mu_{n}\left(1-\alpha_{n}\right)\left\langle z_{n}-x_{n}, j\left(z_{n+1}-x_{n}\right)\right\rangle \\
& +\left(1-\mu_{n}\right)\left\langle f\left(z_{n}\right)-f\left(x_{n}\right), j\left(z_{n+1}-x_{n}\right)\right\rangle \\
& +\left(1-\mu_{n}\right) \alpha_{n}\left\langle x_{n}-T x_{n}, j\left(z_{n+1}-x_{n}\right)\right\rangle \\
= & \mu_{n} \alpha_{n}\left\langle T z_{n+1}-T x_{n}, j\left(z_{n+1}-x_{n}\right)\right\rangle+\mu_{n} \alpha_{n}\left\langle T z_{n}-T z_{n+1}, j\left(z_{n+1}-x_{n}\right)\right\rangle \\
& +\mu_{n}\left(1-\alpha_{n}\right)\left\langle z_{n}-x_{n}, j\left(z_{n+1}-x_{n}\right)\right\rangle \\
& +\left(1-\mu_{n}\right)\left\langle f\left(z_{n}\right)-f\left(x_{n}\right), j\left(z_{n+1}-x_{n}\right)\right\rangle \\
& +\left(1-\mu_{n}\right) \alpha_{n}\left\langle x_{n}-T x_{n}, j\left(z_{n+1}-x_{n}\right)\right\rangle \\
\leq & \mu_{n} \alpha_{n}\left\|z_{n+1}-x_{n}\right\|^{2}+\mu_{n} \alpha_{n}\left\|T z_{n}-T z_{n+1}\right\|\left\|z_{n+1}-x_{n}\right\| \\
& +\mu_{n}\left(1-\alpha_{n}\right)\left\|z_{n}-x_{n}\right\|\left\|z_{n+1}-x_{n}\right\|+\left(1-\mu_{n}\right)\left\|f\left(z_{n}\right)-f\left(x_{n}\right)\right\|\left\|z_{n+1}-x_{n}\right\| \\
& +\left(1-\mu_{n}\right) \alpha_{n}\left\|x_{n}-T x_{n}\right\|\left\|z_{n+1}-x_{n}\right\| .
\end{aligned}
$$

Thus, we have that

$$
\begin{aligned}
\left\|z_{n+1}-x_{n}\right\| \leq & \mu_{n} \alpha_{n}\left\|z_{n+1}-x_{n}\right\|+\mu_{n} \alpha_{n}\left\|T z_{n}-T z_{n+1}\right\| \\
& +\left[\mu_{n}\left(1-\alpha_{n}\right)+\left(1-\mu_{n}\right) \alpha\right]\left\|z_{n}-x_{n}\right\|+\left(1-\mu_{n}\right) \alpha_{n}\left\|x_{n}-T x_{n}\right\| \\
\leq & \mu_{n} \alpha_{n}\left\|z_{n+1}-x_{n}\right\|+\mu_{n} \alpha_{n} \phi\left(\left\|z_{n}-z_{n+1}\right\|\right) \\
& +\left[\mu_{n}\left(1-\alpha_{n}\right)+\left(1-\mu_{n}\right) \alpha\right]\left\|z_{n}-x_{n}\right\|+\left(1-\mu_{n}\right) \alpha_{n}\left\|x_{n}-T x_{n}\right\|,
\end{aligned}
$$

so that

$$
\begin{aligned}
\left\|z_{n+1}-x_{n}\right\| \leq & {\left[1-\frac{(1-\alpha)\left(1-\mu_{n}\right)}{1-\alpha_{n} \mu_{n}}\right]\left\|z_{n}-x_{n-1}\right\|+\left\|x_{n-1}-x_{n}\right\| } \\
& +\frac{\alpha_{n}}{1-\alpha_{n} \mu_{n}} \phi\left(\left\|z_{n}-z_{n+1}\right\|\right)+\frac{\left(1-\mu_{n}\right) \alpha_{n}}{1-\alpha_{n} \mu_{n}}\left\|x_{n}-T x_{n}\right\| .
\end{aligned}
$$

Since the mapping $\widetilde{J_{n}}:=\left[I+\left(\alpha_{n} /\left(1-\mu_{n}\right)\right)(I-T)\right]^{-1}$ is nonexpansive and $x_{n}=\widetilde{J}_{n}\left(f\left(x_{n}\right)\right)$,

$$
\begin{aligned}
\left\|x_{n}-x_{n-1}\right\| & =\left\|\tilde{J}_{n}\left(f\left(x_{n}\right)\right)-x_{n-1}\right\|=\left\|\tilde{J_{n}}\left(f\left(x_{n}\right)\right)-\tilde{J}_{n}\left(f\left(x_{n-1}\right)\right)+\tilde{J}_{n}\left(f\left(x_{n-1}\right)\right)-x_{n-1}\right\| \\
& \leq\left\|f\left(x_{n}\right)-f\left(x_{n-1}\right)\right\|+\left\|\tilde{J_{n}}\left(f\left(x_{n-1}\right)\right)-x_{n-1}\right\| \\
& \leq \alpha\left\|x_{n}-x_{n-1}\right\|+\left\|\tilde{J_{n}}\left(f\left(x_{n-1}\right)\right)-x_{n-1}\right\|,
\end{aligned}
$$


so that

$$
\begin{aligned}
\left\|x_{n}-x_{n-1}\right\| & \leq \frac{1}{1-\alpha}\left\|\tilde{J}_{n}\left(f\left(x_{n-1}\right)\right)-x_{n-1}\right\| \\
& \leq \frac{1}{1-\alpha}|| f\left(x_{n-1}\right)-\left[x_{n-1}+\frac{\alpha_{n}}{1-\mu_{n}}\left(x_{n-1}-T x_{n-1}\right)\right] \| \\
& =\frac{1}{1-\alpha}\left|\frac{\alpha_{n-1}}{1-\mu_{n-1}}-\frac{\alpha_{n}}{1-\mu_{n}}\right|\left\|x_{n-1}-T x_{n-1}\right\| \\
& =\frac{1}{1-\alpha}\left|1-\frac{\alpha_{n}}{1-\mu_{n}} \frac{1-\mu_{n-1}}{\alpha_{n-1}}\right|\left\|f\left(x_{n-1}\right)-x_{n-1}\right\| \\
& =\frac{1}{1-\alpha}\left|\frac{\left(\alpha_{n-1}-\alpha_{n}\right)\left(1-\mu_{n}\right)+\alpha_{n}\left(\mu_{n-1}-\mu_{n}\right)}{\alpha_{n-1}\left(1-\mu_{n}\right)}\right|\left\|f\left(x_{n-1}\right)-x_{n-1}\right\| \\
& \leq \frac{1}{1-\alpha}\left[\frac{\alpha_{n-1}-\alpha_{n}}{\alpha_{n-1}}+\frac{\left|\mu_{n-1}-\mu_{n}\right|}{1-\mu_{n}}\right]\left\|f\left(x_{n-1}\right)-x_{n-1}\right\| .
\end{aligned}
$$

We estimate $\left\|z_{n}-z_{n+1}\right\|$. Let $c:=\sup _{n \geq 1}\left\{\left(1-\mu_{n}\right) / \alpha_{n}\right\}$. Since the sequences $\left\{z_{n}\right\},\left\{x_{n}\right\}$ and the set $\{\phi(t) / t: 0<t<1\}$ are bounded, let $\left\|z_{n}-T z_{n}\right\| \leq M,\left\|x_{n}-T x_{n}\right\| \leq M, \| f\left(z_{n}\right)-$ $z_{n}\|\leq M\| f,\left(x_{n}\right)-x_{n} \| \leq M \forall n \in \mathbb{N}$ and $\sup \{\phi(t) / t: 0<t<1\} \leq M$ for some constant $M>0$. Then

$$
\begin{aligned}
\left\|z_{n+1}-z_{n}\right\| & =\left\|\mu_{n} \alpha_{n}\left(T z_{n}-z_{n}\right)+\left(1-\mu_{n}\right)\left(f\left(z_{n}\right)-z_{n}\right)\right\| \\
& \leq \alpha_{n}\left\|T z_{n}-z_{n}\right\|+\left(1-\mu_{n}\right)\left\|f\left(z_{n}\right)-z_{n}\right\| \\
& \leq\left[\alpha_{n}+\left(1-\mu_{n}\right)\right] M \leq \alpha_{n}(1+c) M,
\end{aligned}
$$

for all $n \in \mathbb{N}$. It follows from (3.5) that

$$
\begin{aligned}
\left\|z_{n+1}-x_{n}\right\| \leq & {\left[1-\frac{(1-\alpha)\left(1-\mu_{n}\right)}{1-\alpha_{n} \mu_{n}}\right]\left\|z_{n}-x_{n-1}\right\|+\frac{1}{1-\alpha}\left[\frac{\alpha_{n-1}-\alpha_{n}}{\alpha_{n-1}}-\frac{\left|\mu_{n}-\mu_{n-1}\right|}{1-\mu_{n}}\right] M } \\
& +\frac{\alpha_{n}}{1-\alpha_{n} \mu_{n}} \phi\left(\alpha_{n}(1+c) M\right)+\frac{\left(1-\mu_{n}\right) \alpha_{n}}{1-\alpha_{n} \mu_{n}} M
\end{aligned}
$$

There exists $N \in \mathbb{N}$ such that $\alpha_{n}(1+c) M<1 \forall n \geq N$. Thus,

$$
\begin{aligned}
\left\|z_{n+1}-x_{n}\right\| \leq & {\left[1-\frac{(1-\alpha)\left(1-\mu_{n}\right)}{1-\alpha_{n} \mu_{n}}\right]\left\|z_{n}-x_{n-1}\right\| } \\
+ & {\left[\frac{1}{1-\alpha}\left(\frac{\alpha_{n-1}-\alpha_{n}}{\alpha_{n-1}}-\frac{\left|\mu_{n}-\mu_{n-1}\right|}{1-\mu_{n}}\right)\right.} \\
& \left.+\frac{\alpha_{n}^{2}(1+c) M}{1-\alpha_{n} \mu_{n}}+\frac{\left(1-\mu_{n}\right) \alpha_{n}}{1-\alpha_{n} \mu_{n}}\right] M, \quad \forall n \geq N .
\end{aligned}
$$


Set $\beta_{n}:=(1-\alpha)\left(1-\mu_{n}\right) /\left(1-\alpha_{n} \mu_{n}\right)$ and $\gamma_{n}:=(1 /(1-\alpha))\left(\left(\alpha_{n-1}-\alpha_{n}\right) / \alpha_{n-1}-\mid \mu_{n}\right.$ $\left.-\mu_{n-1} \mid /\left(1-\mu_{n}\right)\right)+\alpha_{n}^{2}(1+c) M /\left(1-\alpha_{n} \mu_{n}\right)+\left(1-\mu_{n}\right) \alpha_{n} /\left(1-\alpha_{n} \mu_{n}\right)$. Then the inequality

$$
\left\|z_{n+1}-x_{n}\right\| \leq\left(1-\beta_{n}\right)\left\|z_{n}-x_{n-1}\right\|+\gamma_{n} M
$$

follows. By the assumptions on the sequences of numbers $\left\{\alpha_{n}\right\}$ and $\left\{\mu_{n}\right\}$ we find that $\gamma_{n}=o\left(\beta_{n}\right)$. Thus, by Lemma 2.2, $\left\|z_{n+1}-x_{n}\right\| \rightarrow 0$ as $n \rightarrow \infty$, so that

$$
\left\|z_{n}-x_{n}\right\| \leq\left\|z_{n}-z_{n+1}\right\|+\left\|z_{n+1}-x_{n}\right\| \longrightarrow 0
$$

as $n \rightarrow \infty$.

Finally, we show that $\left\|z_{n}-T z_{n}\right\| \rightarrow 0$ as $n \rightarrow \infty$.

Since $\left\|x_{n}-T x_{n}\right\|=\left(1-\mu_{n}\right) / \alpha_{n}\left\|f\left(x_{n}\right)-x_{n}\right\| \leq\left(1-\mu_{n}\right) / \alpha_{n} M \rightarrow 0$ as $n \rightarrow \infty$, and since $T$ is uniformly continuous, we have that

$$
\left\|z_{n}-T z_{n}\right\| \leq\left\|z_{n}-x_{n}\right\|+\left\|x_{n}-T x_{n}\right\|+\left\|T x_{n}-T z_{n}\right\| \longrightarrow 0
$$

as $n \rightarrow \infty$. Hence the proof of Theorem 3.1.

Theorem 3.2. Let $K$ be a nonempty closed convex and bounded subset of a real reflexive Banach space E with a uniformly Gâteaux differentiable norm. Let $T: K \rightarrow K$ be a uniformly continuous pseudocontraction and let $f: K \rightarrow K$ be a contraction map. Suppose that every nonempty closed convex subset of $K$ has the f.p.p. for nonexpansive self-mappings. Let $\left\{z_{n}\right\}$ be a sequence generated from an arbitrary $z_{1} \in K$ by (1.8), where $\left\{\mu_{n}\right\},\left\{\alpha_{n}\right\}$ are real sequences in $(0,1)$ satisfying the same conditions in Theorem 3.1. Then $\left\{z_{n}\right\}$ converges strongly to the fixed point of $T$, which is the unique solution of the variational inequality (2.20).

Proof. By Lemmas 2.3 and 2.5, a sequence $\left\{x_{n}\right\}$ given by $x_{n}=t_{n} T x_{n}+\left(1-t_{n}\right) f\left(x_{n}\right)$, with $t_{n}=\alpha_{n} /\left(1-\mu_{n}+\alpha_{n}\right), n \in \mathbb{N}$ exists and converges strongly to the fixed point of $T$, which is the unique solution of the variational inequality (2.20). From the proof of Theorem 3.1, $\left\|z_{n}-x_{n}\right\| \rightarrow 0$ as $n \rightarrow \infty$. Hence, $\left\{z_{n}\right\}$ converges strongly to the same fixed point of $T$.

Corollary 3.3. Let $K$ be a nonempty closed convex and bounded subset of a real Banach space $E$ with a uniformly Gâteaux differentiable norm. Let $T: K \rightarrow K$ be a uniformly continuous pseudocontraction and let $f: K \rightarrow K$ be a contraction map. Suppose that $K$ has normal structure. Let $\left\{z_{n}\right\}$ be a sequence generated from an arbitrary $z_{1} \in K$ by (1.8), where $\left\{\mu_{n}\right\}$, $\left\{\alpha_{n}\right\}$ are real sequences in $(0,1)$ satisfying the same conditions in Theorem 3.1. Then $\left\{z_{n}\right\}$ converges strongly to the fixed point of $T$, which is the unique solution of the variational inequality (2.20).

Corollary 3.4. Let $K$ be a nonempty closed convex and bounded subset of a real Banach space $E$ with a uniformly Gâteaux differentiable norm and let $T: K \rightarrow K$ be a unformly continuous pseudocontraction. Suppose that every nonempty closed convex subset of $K$ has the f.p.p. for nonexpansive self-mappings. Fix any $w \in K$ and let $\left\{z_{n}\right\}$ be a sequence generated from an arbitrary $z_{1} \in K$ by (1.2), where $\left\{\mu_{n}\right\},\left\{\alpha_{n}\right\}$ are real sequences in $(0,1)$ satisfying the same conditions in Theorem 3.1. Then $\left\{z_{n}\right\}$ converges strongly to the fixed point of $T$, which is the unique solution of the variational inequality (2.20). 
Remarks 3.5. (A) If the map $T$ is assumed to be Lipschitz in the above results then the condition that the set $K$ or the sequence $\left\{z_{n}\right\}_{n}$ be bounded can be dropped. It is proved in [10] that, in this case, the sequence $\left\{z_{n}\right\}_{n}$ is bounded.

(B) It is clear that the conditions on the iteration parameters $\left\{\alpha_{n}\right\},\left\{\mu_{n}\right\}$ in Theorems 3.1, 3.2 and Corollaries 3.3, 3.4 are much simpler than those imposed on the parameters in Theorem 1.1. Examples of real sequences $\left\{\mu_{n}\right\}$ and $\left\{\alpha_{n}\right\}$ that satisfy the conditions (i), (ii), and (iii) of Theorem 3.1 are

$$
\mu_{n}=1-(n+1)^{-1 / 2} \text { and } \alpha_{n}=(n+1)^{-1 / 3},
$$

respectively.

\section{Acknowledgments}

The author thanks the referees for valuable suggestions for the improved version of the paper. Proposition 2.4 and the proofs of Lemmas 2.3 and 2.5 were initially excluded in the first submission.

\section{References}

[1] K. Deimling, Zeros of accretive operators, Manuscripta Mathematica 13 (1974), no. 4, 365-374.

[2] B. Halpern, Fixed points of nonexpanding maps, Bulletin of the American Mathematical Society 73 (1967), 957-961.

[3] T. Kato, Nonlinear semigroups and evolution equations, Journal of the Mathematical Society of Japan 19 (1967), 508-520.

[4] T.-C. Lim, On characterizations of Meir-Keeler contractive maps, Nonlinear Analysis. Theory, Methods \& Applications. Series A: Theory and Methods 46 (2001), no. 1, 113-120.

[5] R. H. Martin Jr., Differential equations on closed subsets of a Banach space, Transactions of the American Mathematical Society 179 (1973), 399-414.

[6] C. H. Morales, On the fixed-point theory for local k-pseudocontractions, Proceedings of the American Mathematical Society 81 (1981), no. 1, 71-74.

[7] C. H. Morales and J. S. Jung, Convergence of paths for pseudo-contractive mappings in Banach spaces, Proceedings of the American Mathematical Society 128 (2000), no. 11, 3411-3419.

[8] A. Moudafi, Viscosity approximation methods for fixed-points problems, Journal of Mathematical Analysis and Applications 241 (2000), no. 1, 46-55.

[9] J. Schu, Approximating fixed points of Lipschitzian pseudocontractive mappings, Houston Journal of Mathematics 19 (1993), no. 1, 107-115.

[10] A. Udomene, Path convergence, approximation of fixed points and variational solutions of pseudocontractions in Banach spaces, submitted to Nonlinear Analysis, TMA.

[11] H.-K. Xu, Iterative algorithms for nonlinear operators, Journal of the London Mathematical Society. Second Series 66 (2002), no. 1, 240-256.

[12] _ Viscosity approximation methods for nonexpansive mappings, Journal of Mathematical Analysis and Applications 298 (2004), no. 1, 279-291.

Aniefiok Udomene: Department of Mathematics, Statistics, \& Computer Science,

University of Port Harcourt, PMB 5323, Port Harcourt, Nigeria

E-mail address: epsilon_ani@yahoo.com 